\title{
The relationship between spiritual well-being and demographic characteristic of students of Qazvin University of Medical Sciences
}

\author{
K. Asgari Ghoncheh"1, H. Hashemnezhad ${ }^{1 r}$, HR. Hajibabaeei ${ }^{1 \longleftarrow}$
}

\footnotetext{
${ }^{1}$ Department of Islamic Studies Resources, School of Islamic and Islamic thought University of Tehran, Tehran, Iran

Corresponding Address: Hamid Reza Hajibabaeei, Islamic and Islamic thought, University of Tehran, Tehran, Iran Tel: +98-28-33334893; Email: hajibabaeei@ut.ac.ir

Received: 7 Jun 2018; Accepted: 30 Jun 2018
}

\section{* Abstract}

Background: Spiritual well-being is considered as an important aspect of heath. It plays an important role in the development of health outcomes.

Objective: This study was conducted to investigate the statues of spiritual well-being of student of Qazvin University of Medical Sciences as the health care providers.

Methods: This descriptive-analytical study was conducted on 307 students which selected from Qazvin University of Medical Science by multi-stage sampling in 2019. The data were collected using Ellison and Paloutzian 20-items spiritual well-being questionnaire. The reliability of questionnaire was examined through cronbach's coefficient alpha approach. Data were analyzed using independent t-test and ANOVA.

Findings: The results showed that the mean of religious well-being was $36.20 \pm 6.84$, existential wellbeing $34.59 \pm 5.82$ and total spiritual scores were moderate $(70.78 \pm 11.33)$. Significant relationships were found between spiritual well-being and field of study ( $\mathrm{P}<0.001)$, level of study $(\mathrm{P}=0.004)$, and housing conditions $(\mathrm{P}<0.001)$.

Conclusion: According to the findings, the level of spiritual well-being among students was moderate which was related to their field, level of study and housing conditions. Due to the impact of these results on the health outcomes of the community, it should stimulate discussion about the necessity of planning to improve the level of students' spiritual well-being according to their demographic variables.

Keywords: Spiritual well-being, Existential well-being, Religion, Demographic factors Citation: Asgari Ghoncheh K, Hashemnezhad H, Hajibabaeei HR. The relationship between spiritual well-being and demographic characteristic of students of Qazvin University of Medical Sciences. J Qazvin Univ Med Sci 2018; 22(3): 50-57.

\footnotetext{
1asgarighoncheh.k@ut.ac.ir

${ }^{2}$ hashemnezhad48@ut.ac.ir

${ }^{3}$ hajibabaeei@ut.ac.ir
} 


\title{
بررسى ارتباط وضعيت سلامت معنوى با متغيرهاى جمعيتشناختى دانشجويان

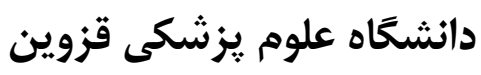

\author{
كريم عسكرى غنجه '، دكتر حسين هاشمنزاد'، دكتر حميدرضا حاجى بابايى'
}

'كروه منابع اسلامى دانشكده معارف و انديشه اسلامى دانشكاه تهران، تهران، ايران

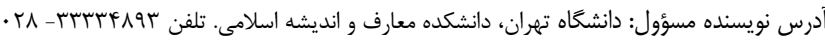

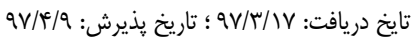

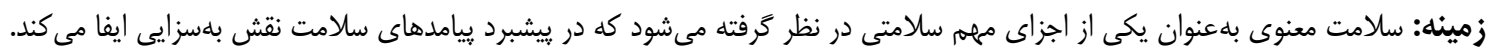

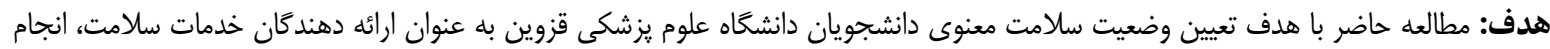

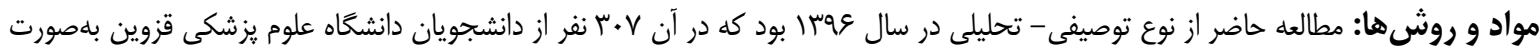

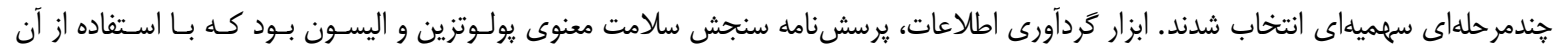

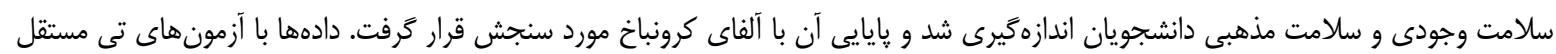

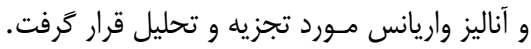

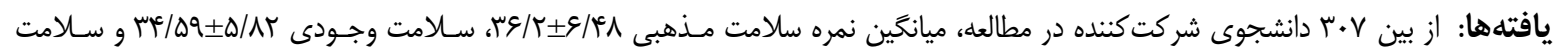

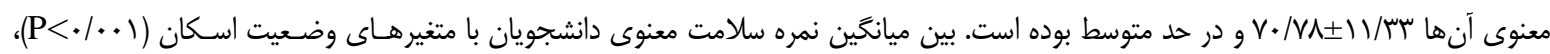

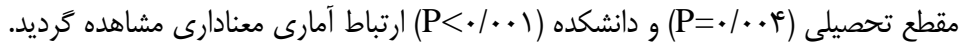

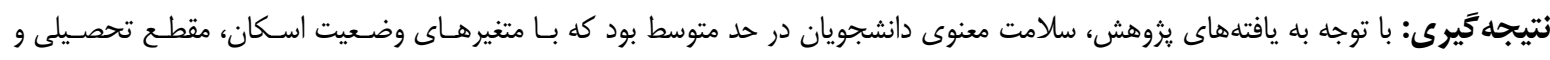

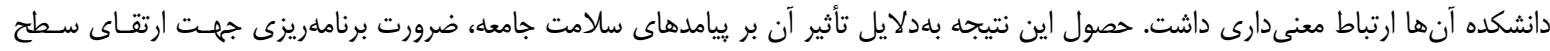

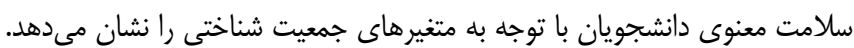

كليدوازهها: سلامت معنوى، سلامت وجودى، مذهب، عوامل جمعيتشناختى

مقدمه:

مطلوبى داشته باشد و در نتيجـهـ بـالاترين سـطح كيفيـت

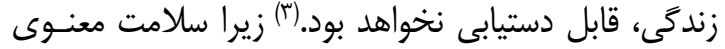
با دارا بودن دو مؤلفه سلامت مذهبى (احساس سلامتى و وانس

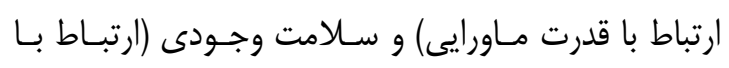

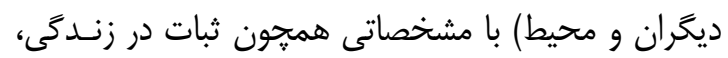

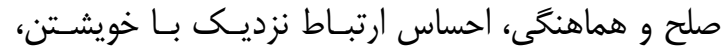

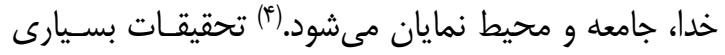

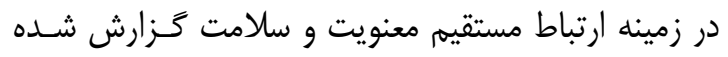

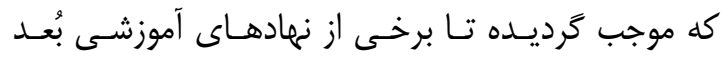
معنوى سلامت را در دستورالعملهاى بـالينى دانشـجويان
مفهوم سلامت از ديـدكًاه سـازمان جهـانى بهداشـت (WHO) جسمى، روانى، اجتماعى و معنوى مى باشد. مطابق تعريف

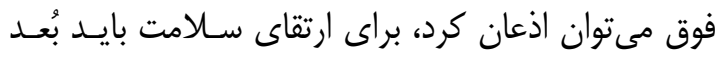

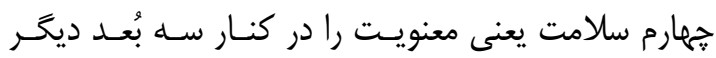

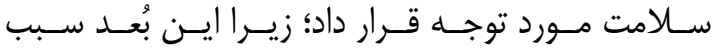

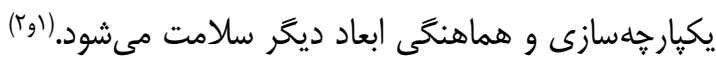

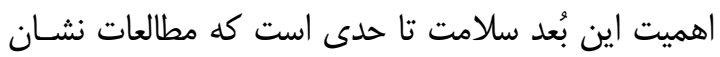
داده است بدون در نظر گرفتن سلامت معنوى، ساير ابعاد

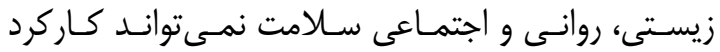


و تاكنون تحقيقى در اين زمينه در دانشگاه علوم يزشـكى

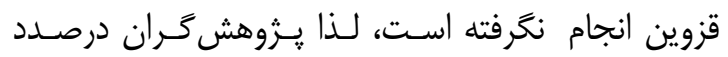

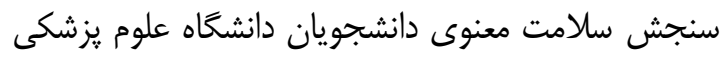

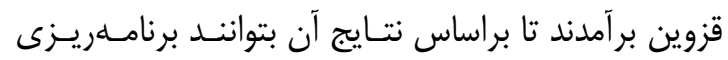

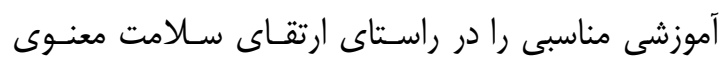
دانشجويان تدوين و طراحى نمايند.

\section{ئمواد و روشها:}

يزوهش حاضر يك مطالعه توصيفى - تحليلى و از نوع همبستخى است كه جامعه يزوهش را دانشجويان دانشعاه

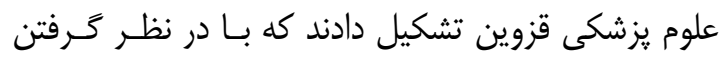

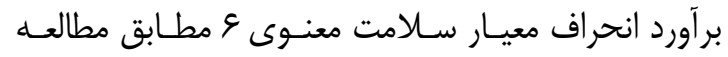

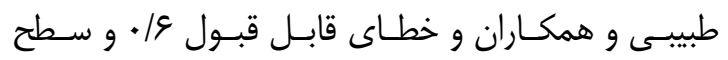

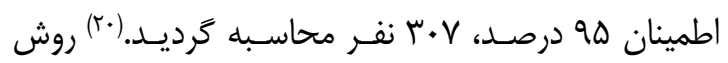

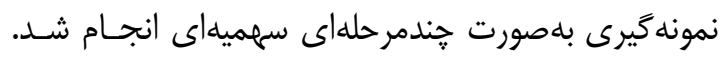

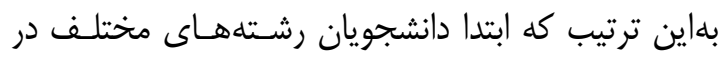

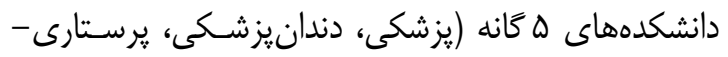

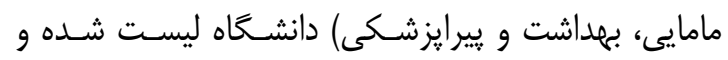
بلهصورت قرعه كشى براساس حجم نمونسه از دانشـكدههــا

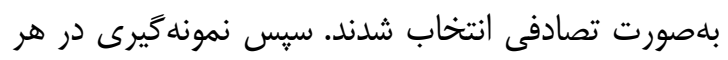

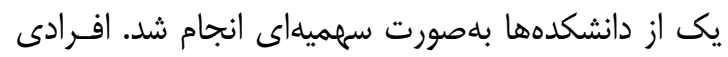

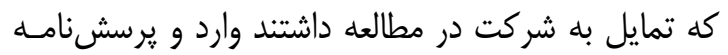
را تكميل نمودند.

ابزار گردآورى اطلاعات شامل دو ابزار بود: 1- يرسشنامه اطلاعات جمعيتششناختى كه شامل؛ سـن، وضعيت تأهل و سطح تحصيلات دانشجويان بود.

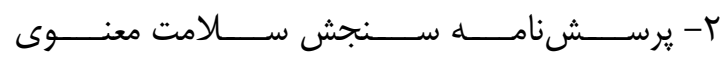
توســــــــ (Spiritual well-being scale; SWBS)

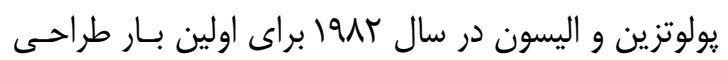

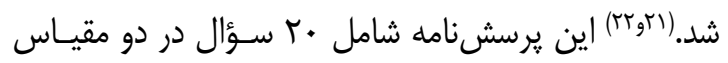

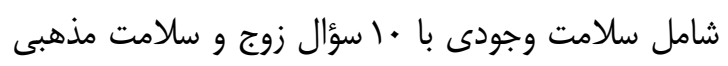

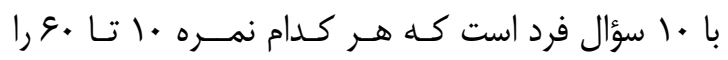

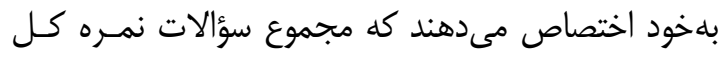
سلامت معنوى را تشكيل مىدهد كه نمره آن بين • ب تـا
خــود جهـت انجـام مراقبـت مبتنـى بـر معنويـت لحـاظ نمايند. (هو جهود

شواهد درباره تأثير آموزش در موضوعات معنويست در دانشجويان نشان داده است كه دانشـويانى كـه در دوره دوره

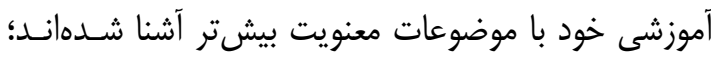
نخرش مثبت كترى به معنويت داشته و كرايش بيشترى در

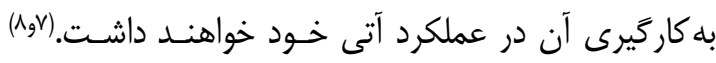

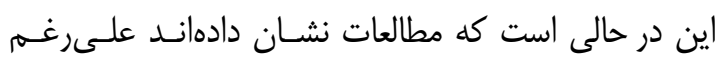

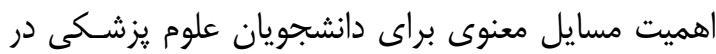

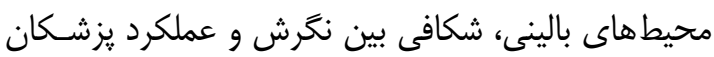

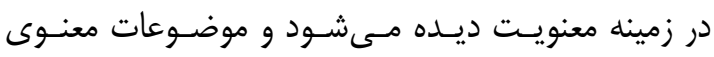

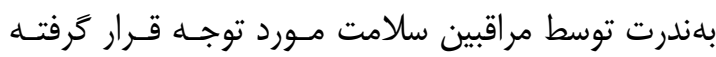

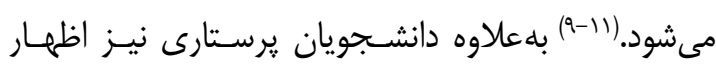

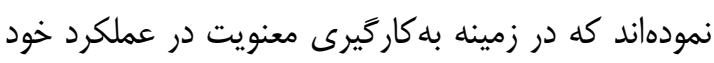

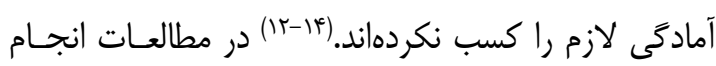

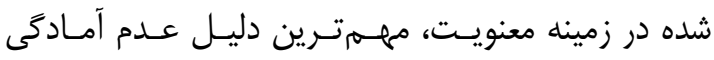

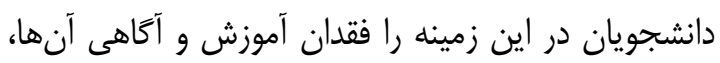

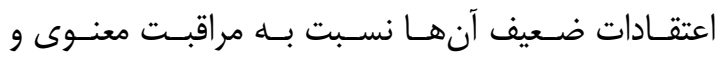

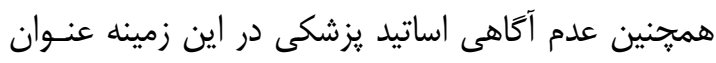

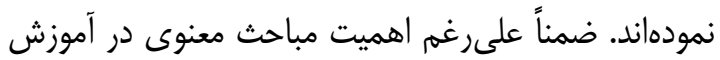

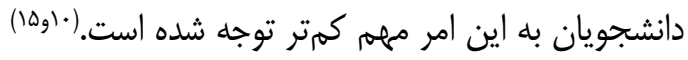

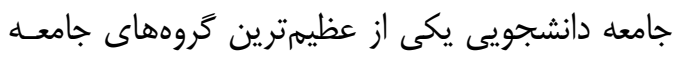

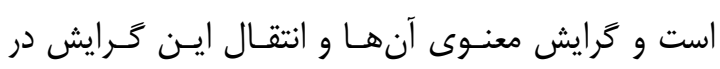

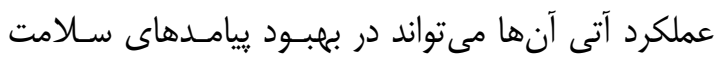

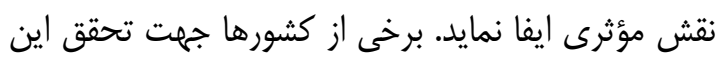

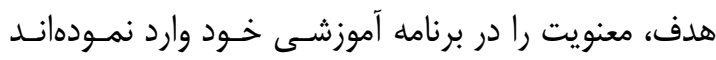

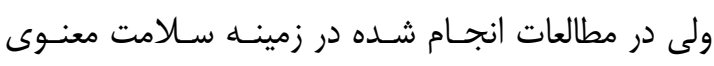

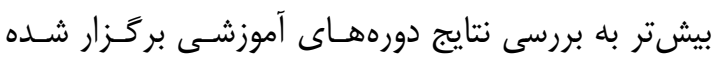

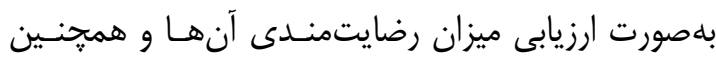

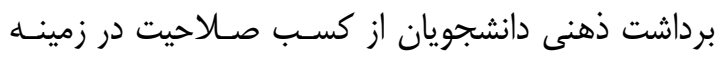

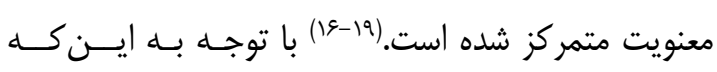

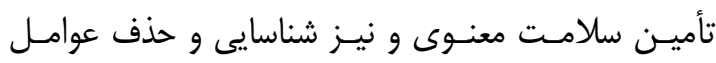

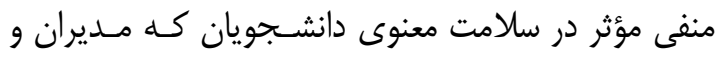

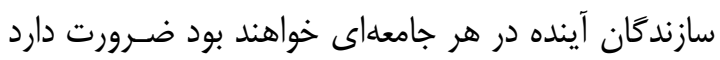


ارتباط بين متغيرهـاى جنـس، وضعيـت تأهـل، وضعيـت

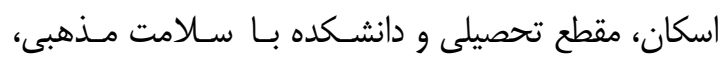

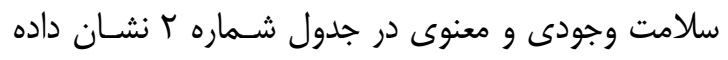

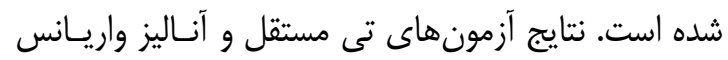

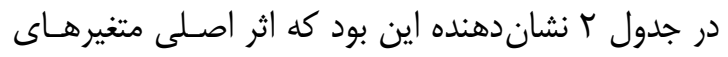

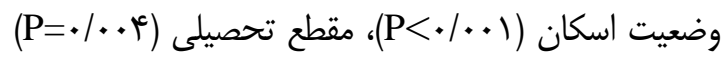

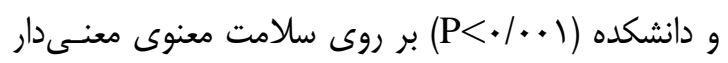

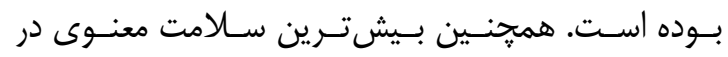

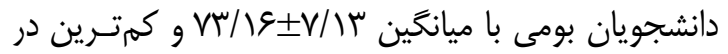

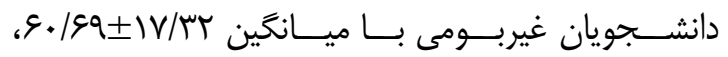
بيشترين سلامت معنوى در مقطع كـاردانى بـا ميـانخين

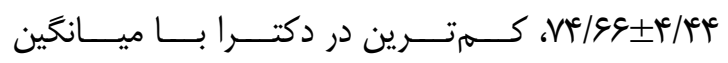
ع

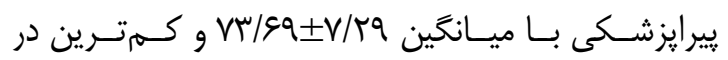

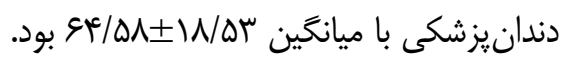

جدول ا - توزيع فراوانى دانشجويان به تفكيك جميت خصوصيات جمعيتشناختى

\begin{tabular}{|c|c|c|c|}
\hline درصد & فراوانى & متغير & هارامتر \\
\hline$r \cdot / v$ & ITD & يسر & \multirow{2}{*}{ جنسيت } \\
\hline$\Delta q / \mu$ & MN & دختر & \\
\hline$V / \Delta$ & r & متأهل & \multirow{2}{*}{ وضعيت تأهل } \\
\hline $9 Y / \Delta$ & rAF & مجرد & \\
\hline$f 1 / V$ & ITA & بومى & \multirow{4}{*}{ وضعيت اسكان } \\
\hline$\Delta / r$ & 19 & غيربومى & \\
\hline$r N / q$ & 10. & خوابكاهى & \\
\hline$r / r$ & M & شخصى & \\
\hline$r / q$ & 9 & كاردانى & \multirow{3}{*}{ مقطع تحصيلى } \\
\hline$\Delta V / V$ & IVV & كارشناسى & \\
\hline$r q / \uparrow$ & $|r|$ & دكترى & \\
\hline$I V / 9$ & DF & يزشكى & \multirow{5}{*}{ دانشكده } \\
\hline$r / \Lambda$ & $9 V$ & دندانيزشكى & \\
\hline $1 \% / V$ & et & يرستارى & \\
\hline$r \cdot / r$ & צ & بهداشت & \\
\hline $18 / 9$ & DI & ييرإيزشكى & \\
\hline
\end{tabular}

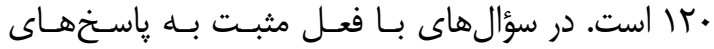

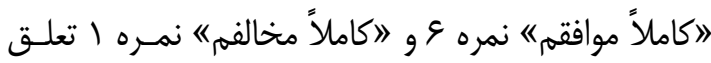

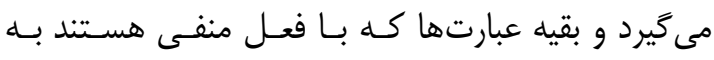

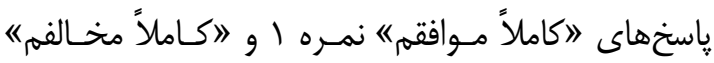
نمره 8 اختصاص مىيابد.

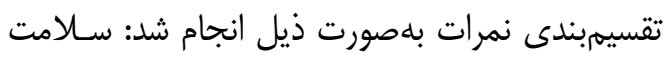

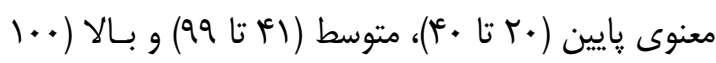

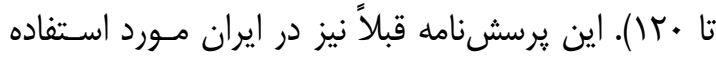

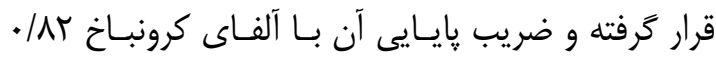

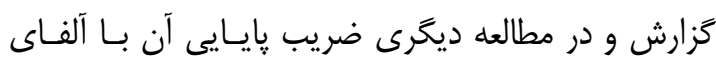

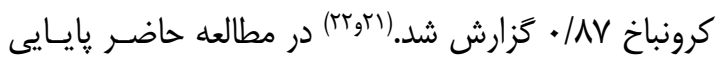

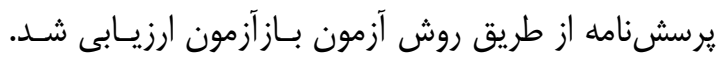

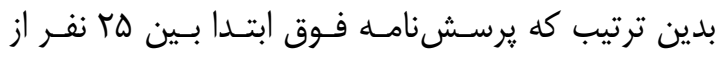

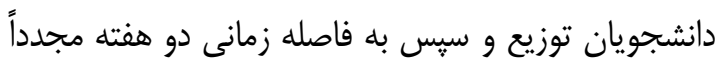

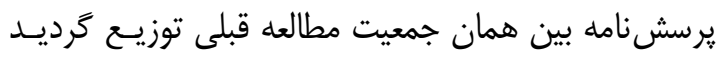

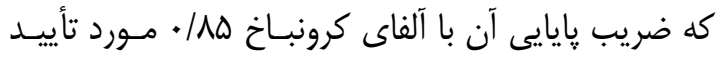

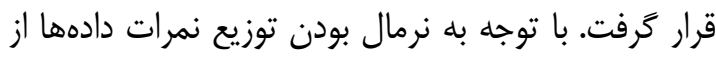

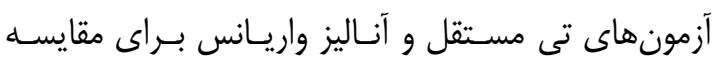

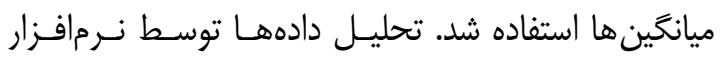

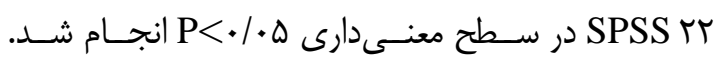

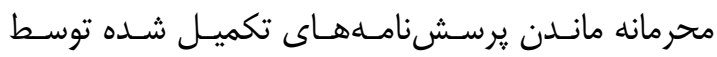

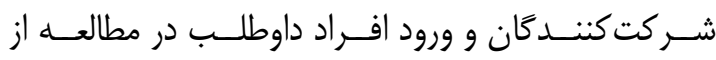
ملاحظات اخلاقى يزوهش حاضر بوده است.

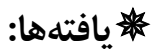

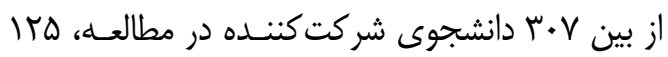

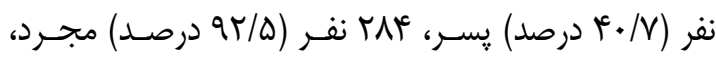

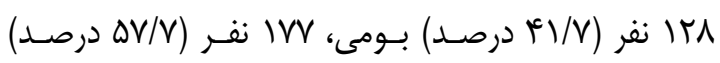

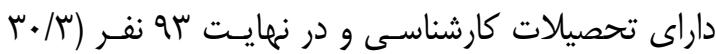

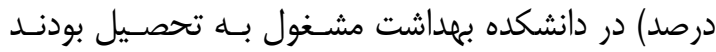

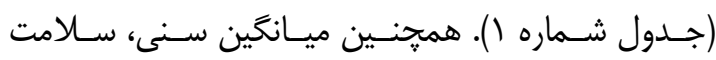
مذهبى، سلامت وجودى و سـلامت معنـوى دانشـجـويان

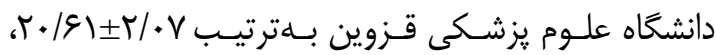
ه 
جدول ץ- مقايسه ميانكين سلامت مذهبى، وجودى و معنوى بر خصوصيات جمعيت شناختى

\begin{tabular}{|c|c|c|c|c|c|c|c|}
\hline \multicolumn{2}{|c|}{ سلامت معنوى } & \multicolumn{2}{|c|}{ سلامت وجودى } & \multicolumn{2}{|c|}{ سلامت مذهبى } & \multirow[t]{2}{*}{ متغير } & \multirow[t]{2}{*}{ 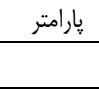 } \\
\hline سطح معنىدارى & انحراف معيار 土 ميانكين & سطح معنىدارى & انحراف معيار 土 ميانكين & سطح معنىدارى & انحراف معيار 土 ميانكَين & & \\
\hline \multirow{2}{*}{ ת } & $99 / \Lambda \& \pm|r / 4|$ & \multirow{2}{*}{$\cdot / v \cdot 9$} & $\mu F / V \Psi \pm \varepsilon / \mu \Delta$ & \multirow{2}{*}{$.1 \cdot 10$} & $r \Delta / / r \pm \varepsilon / 91$ & טֶן & \multirow{2}{*}{ جنسيت" } \\
\hline & $V T / F \Psi \pm 1 \cdot / \Delta T$ & & 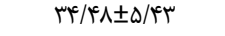 & & $r \varepsilon / 9 \Delta \pm \varepsilon / \cdot 1$ & دختر & \\
\hline \multirow{2}{*}{$\cdot 1 \cdot 1 \Delta$} & $9 \Delta / \cdot \wedge \pm \mid \varepsilon / 1 Y$ & \multirow{2}{*}{$.1 \cdot .1$} & $r \cdot / V \wedge \pm \varepsilon / Q \varepsilon$ & \multirow{2}{*}{.$/ W M$} & $m e / r \cdot \pm q / F \wedge$ & متأهل & \multirow{2}{*}{ تأهل" } \\
\hline & $V I / T \Delta \pm I \cdot / V G$ & & $r r / \wedge q \pm \Delta / q r$ & & T & مجرد & \\
\hline \multirow{4}{*}{$<\cdot / \cdot+1$} & $V \Psi / \wedge \varepsilon \pm V / / r$ & \multirow{4}{*}{. } & $r \Delta / F^{*} V \pm \Psi / \cdot q$ & \multirow{4}{*}{$<\cdot 1 \cdot \cdot 1$} & $r V / \varsigma q \pm r / V$. & بومى ل & \multirow{4}{*}{ اسكان "* } \\
\hline & $9 \cdot 199 \pm 1 V / \pi T$ & & $r q / v \Delta \pm N / I V$ & & $r \cdot / q q \pm q / q q$ & غير بومى & \\
\hline & $V \cdot 1 \cdot \Delta \pm 1 r / q r$ & & $M F / r T \pm \xi / \varepsilon$. & & $r \Delta / \Upsilon^{\top} \pm V / \cdot 1$ & خوابكاهى & \\
\hline & $9 \Lambda / r \Lambda \pm N / \Lambda)$ & & $r F / Q T \pm r / \Delta T$ & & Tr/ז\& $\pm \xi / T \Delta$ & شخصى & \\
\hline \multirow{3}{*}{..$+ t$} & $V \varphi / \& \oint \pm Y / 4 Y$ & \multirow{3}{*}{. $/ . T V$} & $r V / r T \pm T / V V$ & \multirow{3}{*}{ H. r } & $r V / F \Psi \pm \Psi / V \Lambda$ & كاردانى & \multirow{3}{*}{ "حصيلى" مقطع. } \\
\hline & $V T / T V \pm V / \& Y$ & & $r \Delta / / \Delta \pm \Gamma / \Delta q$ & & $r V / R r \pm \Psi /$ LV & كارشناسى & \\
\hline & $9 N / \backslash \Lambda \pm \mid Q / / F$ & & $r / \Delta V \pm V / r F$ & & $r F / \xi 1 \pm \Lambda / V \Delta$ & دكترى & \\
\hline \multirow{5}{*}{$<\cdot / \cdot \cdot 1$} & $V Y / \& \Delta \pm V / F 1$ & \multirow{5}{*}{$.1 . .1$} & 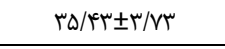 & \multirow{5}{*}{$<\cdot 1 \cdot \cdot 1$} & $r V / r r \pm g / r \Lambda$ & يزشكى & \multirow{5}{*}{ دانشكده" } \\
\hline & $G F / \Delta \Lambda \pm I N / \Delta T$ & & $\mathrm{rT} / \cdot \mathrm{V} \pm \mathrm{N} / \wedge \mathrm{q}$ & & $r Y / \Delta I \pm q / \Lambda \varepsilon$ & دندانيزشكى & \\
\hline & $v \cdot / 4 \cdot \pm 11 / \cdot 1$ & & $\mu \varphi / \cdot r \pm \varepsilon / r \Lambda$ & & $|\varepsilon / \cdot \vee \pm \varepsilon| \cdot 1$ & يرستارى & \\
\hline & $V T / V E \pm \Delta / 19$ & & $r \Delta / r \Lambda \pm r / \Delta F$ & & $r V / \wedge \wedge \pm r / q q$ & بهداشت & \\
\hline & $V / / \varepsilon q \pm V / r q$ & & $r \Delta / q F \pm r / \Delta f$ & & $r V / V \Delta \pm \Psi / M$ & ييرايزشكى & \\
\hline
\end{tabular}

\section{אبحث و نتيجه}

كرده بودند. (T/) همجنين در مطالعـه هياسـائو و همكـاران

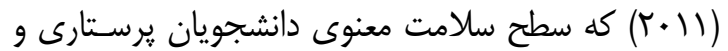

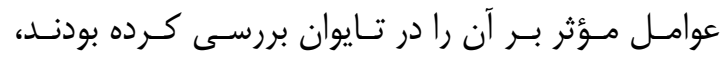

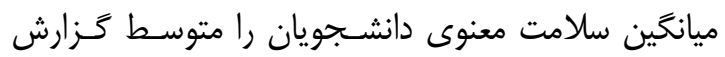

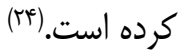
در مطالعه حاضر بيشترين نمره سـلامت معنـوى در

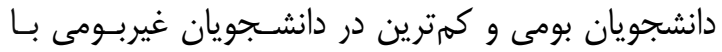

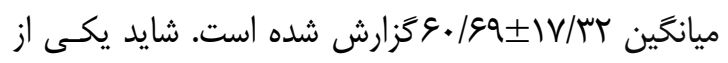

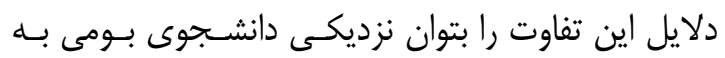

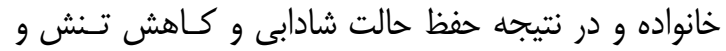

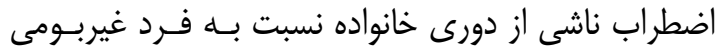

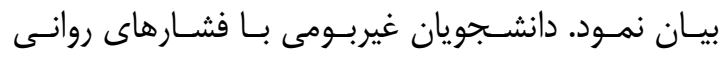

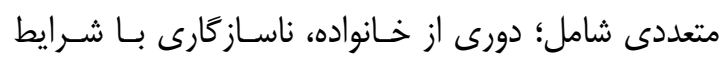

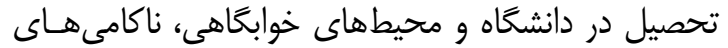
تحصيلى و ... مواجه مىشوند. در مطالعه توان و همكاران

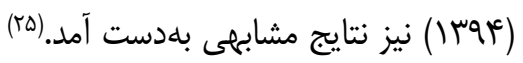

با توجه به اهميت بُعد سلامت معنوى و اثر يكيارجيكى

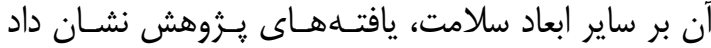
ميانخين سلامت معنوى دانشجويان در سطح متوسط قرار

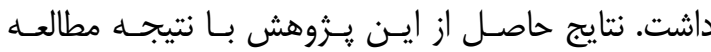

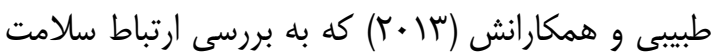
معنوى و ييشرفت تحصيلى دانشجويان يزشـكى دانشـاه

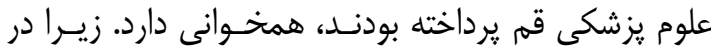

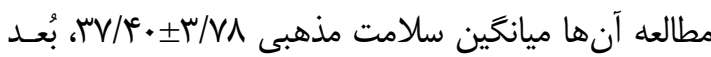

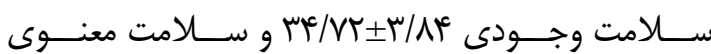
دانشـجويان r+/

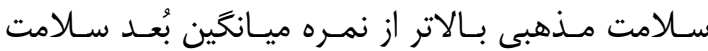

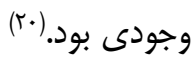

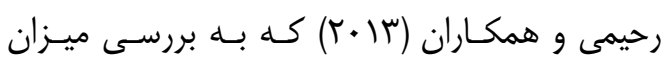
سلامت معنوى دانشجويان يرسـتارى - مامـايى دانشـعاه

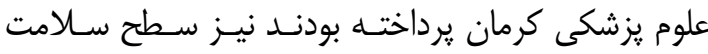

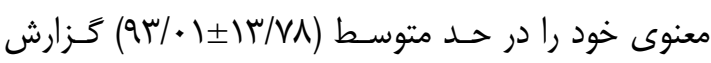


از محدوديتهاى مطالعه مى تـوان بـهـ عـدم در نظـر

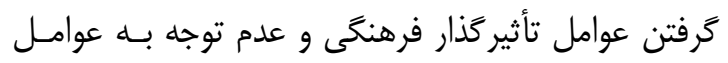

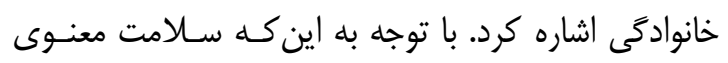
دانشجويان در سطح متوسط قـرار داشـت، بايــد اقـدامات

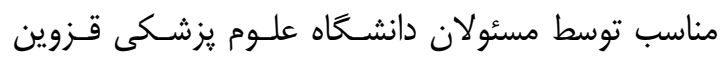

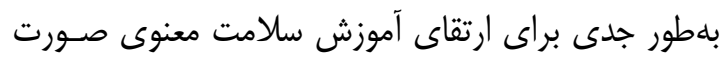

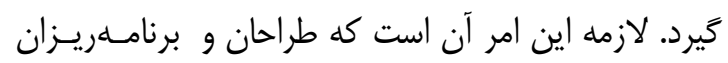

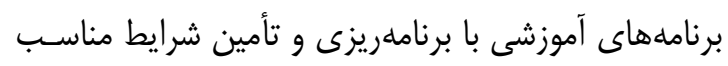

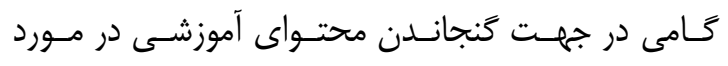

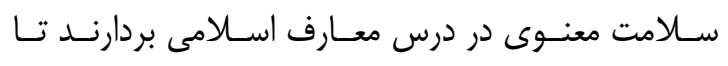

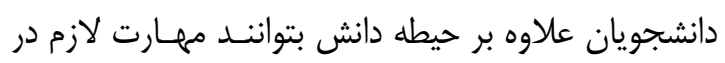
اين زمينه را كسب نمايند.

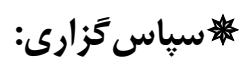

اين مقاله در راستاى انجام بايان نامـهـ مقطع دكتــاى

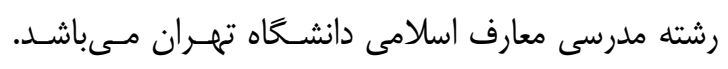

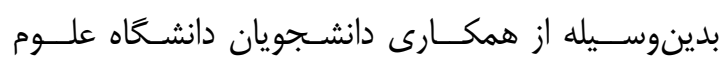

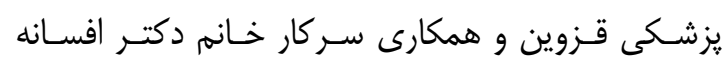

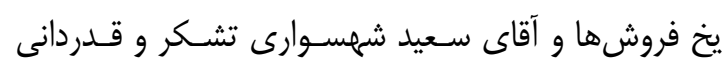

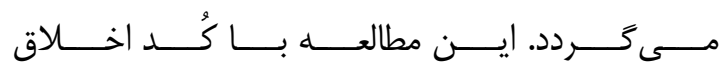
قد در دانشخاه علوم يزشكى

$$
\text { قزوين ثبت شده است. }
$$

\section{: مراجع:}

1. Allahbakhshian M, Jaffarpour M, Parvizy S, Haghani H. A survey on relationship between spiritual wellbeing and quality of life in multiple sclerosis patients. J Zahedan Univ Med Sci 2010; 12(3): 29-33. [In Persian]

2. Kashfi SM, Yazdankhah M, Babaei Heydarabadi A, Khani Jeihooni A, Tabrizi R. The relationship between religious attitude and mental health in students of Shiraz University of Medical Sciences. J Res Religion Health 2015; 1(3): 33-40. [In Persian]
نتايج مطالعه حاضر نشان داد بين متغيرهاى وضـعيت

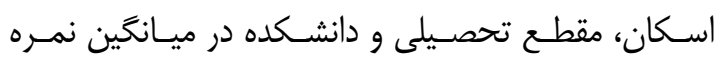

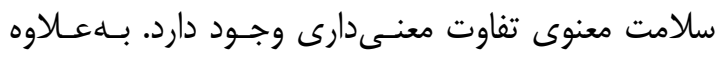

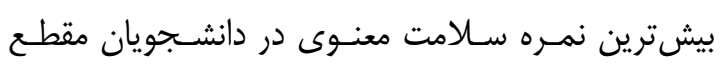

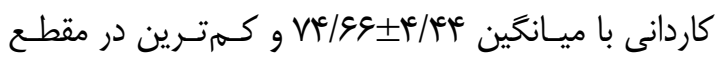

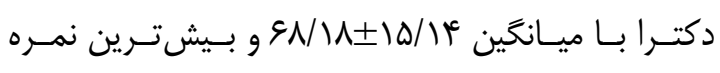

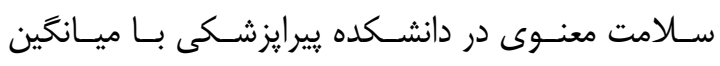

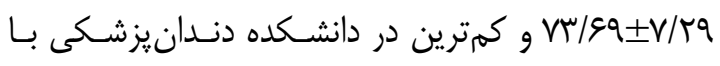

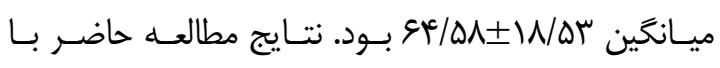

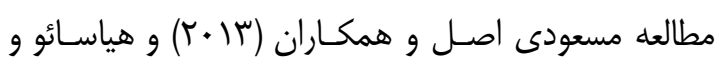

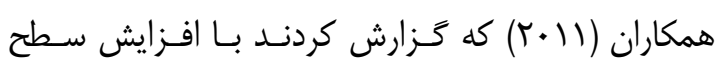

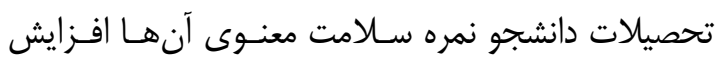

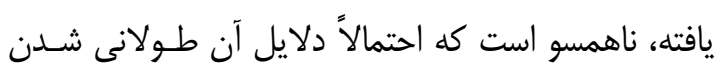

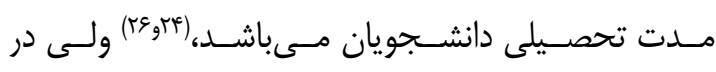

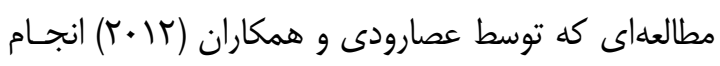

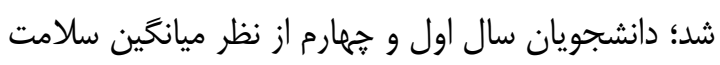

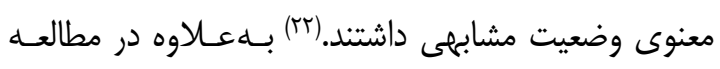

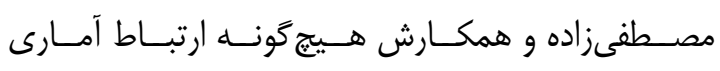

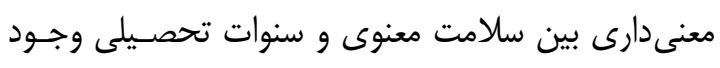
نداشت. (TV) همان طور كه در بالا ذكر شد، نتيجه ديخر اين مطالعه

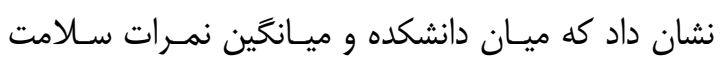

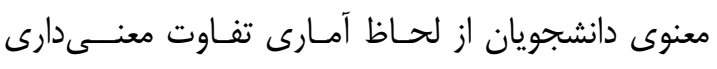

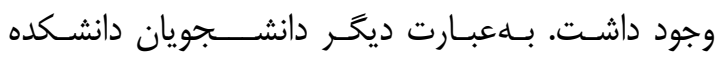

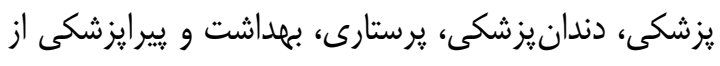

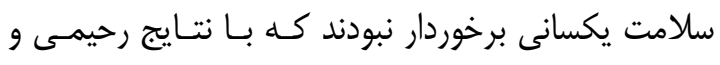

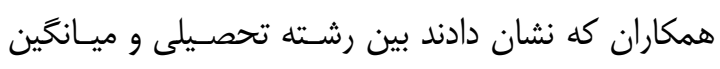

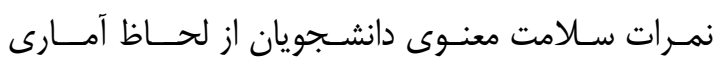

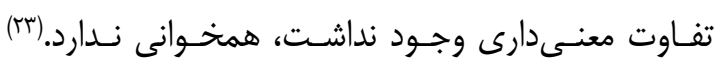

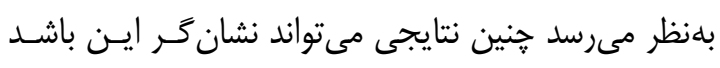

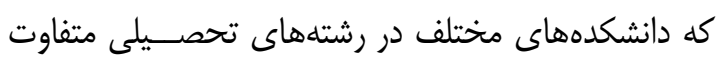

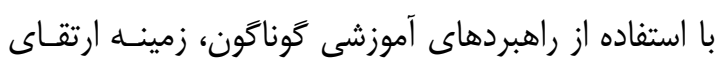

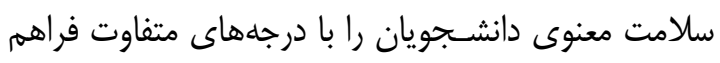
نمايند. 
3. Jadidi A, Farahaninia M, Janmohammadi $\mathrm{S}$, Haghani $\mathrm{H}$. The relationship between spiritual well-being and quality of life among elderly people residing in Kahrizak Senior House. Iran J Nurs 2011; 24(72): 48-56. [In Persian]

4. Marzban S, Babaei Heydarabadi A, Rahimi E, Vejdani M, Shokri M. Spiritual health status in students of Shahid Beheshti University and Shahid Beheshti University of Medical Sciences. J Res Religion Health 2016; 2(4): 17-24. [In Persian]

5. Koenig HG. Religion, spirituality, and health: the research and clinical implications. ISRN Psychiatry 2012; 2012: 278730. doi: 10.5402/2012/278730.

6. Moreira-Almeida A, Pinsky I, Zaleski M, Laranjeira R. Religious involvement and sociodemographic factors: a Brazilian national survey. Rev Psiq Clin 2010; 37(1): 12-5.

7. Luckhaupt SE, Yi MS, Mueller CV, Mrus JM, Peterman AH, Puchalski CM, et al. Beliefs of primary care residents regarding spirituality and religion in clinical encounters with patients: A study at a midwestern US teaching institution. Acad Med 2005; 80(6): 560-70.

8. Saguil A, Fitzpatrick AL, Clark G. Is evidence able to persuade physicians to discuss spirituality with patients? J Relig Health 2011; 50(2): 289-99. doi: 10.1007/ s10943-010-9452-6.

9. Lucchetti G, Lucchetti AG, Badan-Neto AM, Peres PT, Peres MF, Moreira-Almeida A, et al. Religiousness affects mental health, pain and quality of life in older people in an outpatient rehabilitation setting. J Rehabil Med 2011; 43(4): 316-22. doi: 10.2340/ 16501977-0784.

10. Mariotti LG, Lucchetti G, Dantas MF,
Banin VB, Fumelli F, Padula NA. Spirituality and medicine: views and opinions of teachers in a Brazilian medical school. Med Teach 2011; 33(4): 339-40.

11. Monroe MH, Bynum D, Susi B, Phifer N, Schultz L, Franco M, et al. Primary care physician preferences regarding spiritual behavior in medical practice. Arch Intern Med 2003; 163(22): 2751-6. doi:10.1001/ archinte.163.22.2751.

12. Chandramohan S, Bhagwan R. Spirituality and spiritual care in in the context of nursing education in South Africa. Curationis 2015; 38(1): 1-15. doi: 10.4102/ curationis.v38i1.1471.

13. Espinha DC, de Camargo SM, Silva SP, Pavelqueires S, Lucchetti G. Nursing students' opinions about health, spirituality and religiosity. Rev Gaucha Enferm 2013; 34(4): 98-106.

14. Tomasso Cde S, Beltrame IL, Lucchetti G. Knowledge and attitudes of nursing professors and students concerning the interface between spirituality, religiosity and health. Rev Latino-Am Enfermagem 2011; 19(5): 1205-13. doi: 10.1590/S010411692011000500019.

15. Vermandere M, De Lepeleire J, Smeets L, Hannes K, Van Mechelen W, Warmenhoven F, et al. Spirituality in general practice: a qualitative evidence synthesis. Br J Gen Pract 2011; 61(592): e749-60. doi: 10.3399/ bjgp11X606663.

16. Anandarajah G, Stumpff J. Attitudes, knowledge and skills of clerkship medical students regarding spirituality and medicine. Ann Behav Sci Med Educ 2005; 11(2): 85-90. 17. Barnett KG, Fortin AH 6th. Spirituality and medicine. A workshop for medical students and residents. J Gen Intern Med 
2006; 21(5): 481-5. doi: $10.1111 /$ j.15251497.2006.00431.x

18. Lucchetti G, Lucchetti AL, Puchalski CM. Spirituality in medical education: global reality? J Relig Health 2012; 51(1): 3-19. doi: 10.1007/s10943-011-9557-6.

19. Puchalski CM. Spirituality and medicine: curricula in medical education. J Cancer Educ 2006; 21(1): 14-8.

20. Tabibi M, Ahmari Tehran H, Soltani Arabshahi SK, Heidari S, Abdi Z, Safaeipour $\mathrm{R}$. The association between spiritual health and academic achievement in medical students of Qom University of Medical Sciences, 2011. J Qom Univ Med Sci 2013; 7(2): 72-8. [In Persian]

21. Habibi A, Savadpour MT. Spiritual wellbeing in cancer patients under chemotherapy. J Health Care 2011; 13(3): 16-21. [In Persian] 22. Assarroudi A, Jalilvand MR, Oudi D, Akaberi A. The relationship between spiritual well-being and life satisfaction in the nursing staff of Mashhad Hasheminezhad Hospital (2011). Mod Care J 2012; 9(2): 156-62. [In Persian]
23. Rahimi N, Nouhi E, Nakhaee N. Spiritual well-being and attitude toward spirituality and spiritual care in nursing and midwifery students. Iran J Nurs 2013; 26(85): 55-65. [In Persian]

24. Hsiao YC, Chiang HY, Chien LY. An exploration of the status of spiritual health among nursing students in Taiwan. Nurse Educ Today 2010;30(5): 386-92. doi: 10. 1016/j.nedt.2009.05.001.

25. Tavan H, Taghinejad H, Sayehmiri K, Yary Y, khalafzadeh A, Fathizadeh H, et al. Spiritual health of nursing students. Islam Health J 2015; 2(1): 26-32. [In Persian]

26. Masoudi Asl I, Rajabi Vasokolaee G, Goudarzi L, Raadabadi M, Eskandari AH. The evaluation of relationship between mental health and spiritual health of students at Tehran University of Medical Sciences, 2013. Teb va Tazkiyeh 2015; 23(3): 55-66. [In Persian]

27. Asadzadeh F, Mostafazadeh F, Sadeghi S. A survey of the motivation of nursing students toward their field of study selection. J Health Care 2012; 14(1-2): 9-15. [In Persian] 